CERN-EP-2000-031

February 16, 2000

\title{
A facility for the test of large area muon chambers at high rates
}

\author{
S. Agosteo ${ }^{1)}$, S. Altieri ${ }^{2)}$, G. Belli ${ }^{2)}$, A. Bonifas ${ }^{3)}$, V. Carabelli ${ }^{2)}$, L. Gatignon ${ }^{3)}$, \\ N. Hessey ${ }^{4) 9)}$, M. Maggi ${ }^{5)}$, J.-P. Peigneux ${ }^{6)}$, H. Reithler ${ }^{7) 8)}$, M. Silari ${ }^{3)}$, P. Vitulo ${ }^{2)}$ \\ and M. Wegner $\left.{ }^{7}\right)$
}

Submitted to Nucl. Instr. and Methods A

\begin{abstract}
Operation of large area muon detectors at the future Large Hadron Collider (LHC) will be characterized by large sustained hit rates over the whole area, reaching the range of $\mathrm{kHz} / \mathrm{cm}^{2}$. We describe a dedicated test zone built at CERN to test the performance and the aging of the muon chambers currently under development. A radioactive source delivers photons causing the sustained rate of random hits, while a narrow beam of high energy muons is used to directly calibrate the detector performance. A system of remotely controlled lead filters serves to vary the rate of photons over four orders of magnitude, to allow the study of performance as a function of rate.
\end{abstract}

1) Dip. di Ingegneria Nucleare, Politecnico di Milano, via Ponzio 34/3, I-20133 Milano, Italy

2) I.N.F.N. Sezione di Pavia and Dip. di Fìsica Nucleare e Teòrica, Università di Pavia, Via Bassi 6, I-27100 Pavia, Italy

3) CERN, CH-1211 Geneva 23, Switzerland

4) Universität München, D-80333 München, Germany

5) I.N.F.N. Sezione di Bari and Dip. Interateneo di Fìsica, Università di Bari, Via Amendola 173, I-70126 Bari, Italy

6) LAPP, BP 110, F-74941 Annecy-le-Vieux, France

7) III. Physikalisches Institut A, RWTH Aachen, D-52056 Aachen, Germany

8) On temporary leave of absence to CERN, CH-1211 Geneva 23, Switzerland

9) Present address: NIKHEF, Kruislaan 409, NL-1098SJ Amsterdam, The Netherlands 
Recently the construction of a $14 \mathrm{TeV}$ proton-proton collider at CERN (Large Hadron Collider, LHC) [1] and of four large detectors $[2,3,4,5,6,7,8]$ for its exploitation were approved. The main focus of the research at this energy is on very rare events - e.g. some signatures for the Higgs particle are expected at the level of $10^{-14}$. Therefore the collider is designed for a high luminosity of $10^{34} \mathrm{~cm}^{-2} \mathrm{~s}^{-1}$, with beam crossings as close as $25 \mathrm{~ns}$. This translates into multiple interactions per beam crossing and, taking into account the average multiplicity of particles per interaction, into a total production of about 1,400 particles at the center of the detector every $25 \mathrm{~ns}$, most of them showering in the calorimeter section of the detector. The large area muon chambers (about 5 to $20 \mathrm{~m}^{2}$ each) surrounding the calorimeter will be flooded by sustained hit rates ranging from about $1 \mathrm{~Hz} / \mathrm{cm}^{2}$ to several $10^{3} \mathrm{~Hz} / \mathrm{cm}^{2}$. In their majority these hits stem from secondary reactions of low energy (about a few $\mathrm{MeV}$ ) neutrons producing a photon that converts inside the chamber. These neutrons emerge from the calorimeter, but in part also from the shielding of the beam lines.

Muon chambers are usually tested with cosmic rays, i.e. over the whole area but at very low rate, or at test beams for high rates. Such test beams illuminate typically an area of $10 \times 10 \mathrm{~cm}^{2}$, at less than $20 \%$ duty cycle. Only less than $0.1 \%$ of the chamber area is thus exposed, and furthermore the detector can recover during the long pause after each spill of particles. In order to be able to study performance and aging effects under realistic LHC-like conditions, a dedicated test beam featuring sustained, uniform and adjustable high rates over large areas was needed.

We describe here a facility meeting these requirements, which was built at CERN in 1997 and has already been extensively used (see e.g. [4]). Since it was not achievable with a muon beam alone, a composite solution was adopted. A radioactive photon source delivering the desired rate of random hits is complemented by a low intensity narrow beam of high energy muons, which give calibration measurements with tracks.

\section{The Gamma Irradiation Facility}

The Gamma Irradiation Facility (GIF) is a test area in which large area high-energy particle detectors are exposed to a particle beam in the presence of an adjustable high background flux of photons, simulating the background conditions that these detectors will suffer in their operating environment at the Large Hadron Collider at CERN. The GIF is situated in the SPS West Area at the downstream end of the X5 test beam. The zone is surrounded by a concrete wall, $8 \mathrm{~m}$ high and $80 \mathrm{~cm}$ thick. Access is possible through three entry points, namely two access doors for personnel and one large gate for material. A crane allows installation of heavy equipment in the area. A schematic layout of the GIF zone is shown in Fig. 1. The photons are produced by a strong radioactive source, which we will call the irradiator, see Fig. 2, 3, 4 . It is installed in the upstream part of the zone, $5 \mathrm{~m}$ away from the end, and housed inside a lead container, which includes a precisely shaped collimator, designed to permit irradiation of a $6 \mathrm{~m} \times 6 \mathrm{~m}$ area at $5 \mathrm{~m}$ distance from the source. A thin lens-shaped lead filter, mounted at the exit face of the source housing, renders the outcoming flux uniform in the vertical plane orthogonal to the beam direction. At $4 \mathrm{~m}$ distance from the source the flux of $662 \mathrm{keV}$ photons on the axis is $0.86 \times 10^{5} \mathrm{~cm}^{-2} \mathrm{~s}^{-1}$. The conversion probability in a single cell of gas-filled muon detectors is typically in the range of $2 \%$ to $0.3 \%$.

The control of the photon rate is achieved by further lead filters, which allow the maximum rate to be limited, and to vary the rate within a range of four orders of magnitude.

A second irradiation area is available at 90 degrees to the main axis. This area is defined by a separate collimator which allows irradiation of detectors with a high flux over a smaller area, e.g. crystal calorimeters. It can be activated or isolated by means of a separate shutter. If opened, this channel provides a flux of $4.4 \times 10^{6} \mathrm{~cm}^{-2} \mathrm{~s}^{-1}$ photons at $1 \mathrm{~m}$ distance from the source. The two facilities can thus operate separately or in parallel. The collimators and the lateral shutter are shown schematically in Fig. 2. 


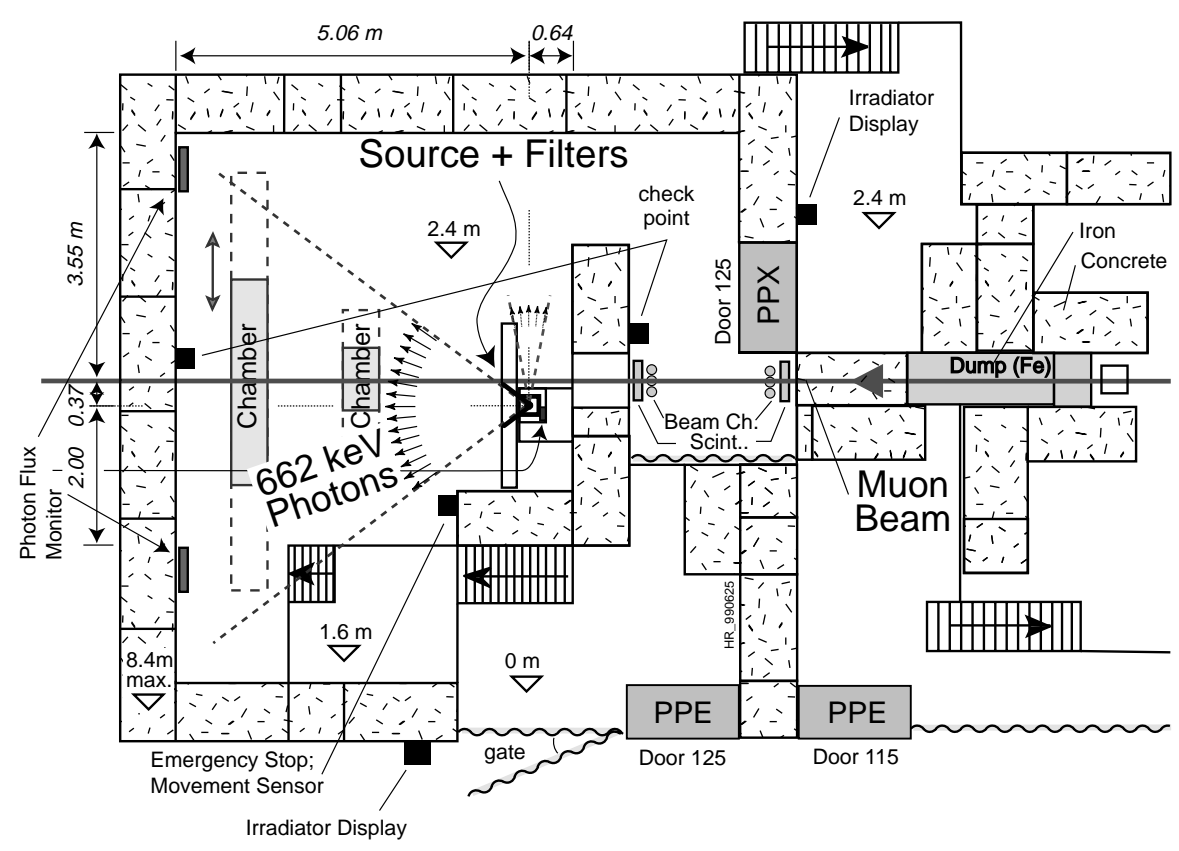

Figure 1: Layout of the test beam zone called X5c GIF at CERN. Photons from the radioactive source flood the detectors, producing a sustained high rate of random hits over the whole area. Calibration with tracks is done with a narrow beam of high energy muons. When displacing the detector transversally to scan different points with the muon beam, the photon flux still encircles the full detector area.

\subsection{Photon source}

The Gamma irradiator is housed in a rectangular container, $400 \mathrm{~mm}$ wide and $900 \mathrm{~mm}$ high, shown in more detail in Fig. 3. The active element (Fig. 4) is a radioactive ${ }^{137} \mathrm{Cs}$ source ${ }^{1)}$ with a half-life of 30 years, the strength of which was measured on the 5th March 1997 to be $740 \mathrm{GBq}$ (implying e.g. $695 \mathrm{GBq}$ in December 1999), which emits a $662 \mathrm{keV}$ photon in $85 \%$ of the decays. All numbers given here refer to the strength of $740 \mathrm{GBq}$. The ${ }^{137} \mathrm{Cs}$ isotope was preferred above ${ }^{60} \mathrm{Co}$ because of its long half-life of 30 years and hence less variation of the photon flux over the expected lifetime of this facility. Also its lower photon energy $(662 \mathrm{keV})$ leads to a twice higher conversion efficiency in the detectors tested. A lead shield of $140 \mathrm{~mm}$ thickness serves as radiobiological protection ${ }^{2)}$. The lead is contained in a $5 \mathrm{~mm}$ thick steel envelope. The principal collimator hole provides a pyramidal aperture of $74^{\circ} \times 74^{\circ}$ solid angle. This provides a photon flux in a pyramidal volume of $5 \mathrm{~m}$ maximum length along the axis. A commercial pneumatic system raises the source to the irradiation position, or lowers it to the shielded position (Fig. 3). In case of pressure drop, the source falls naturally, by its own weight, into its protection container. The components of the protection system add a further $2.3 \mathrm{~mm}$ of stainless steel around the actual source, also in the direction of the main aperture, thus reducing the activity to about $88.5 \%$ of its nominal value.

\subsection{Adjustable photon flux}

The pointlike radiation source delivers a flux of photons of fixed rate, which is uniform over a spherical surface. In contrast to this, for the systematic test of flat large area detectors the rate should be uniform over a plane and should be adjustable; some detectors with limited rate capability may even need a safe protection against too high rates. These three features are all implemented at GIF, with the help of specially shaped lead filters. Each filter is mounted

\footnotetext{
1) Radiactive material from Amersham-Buchler GmbH \& Co KG, Gieselweg 1, D-38110 Braunschweig, Germany.

2) Protection system from STS GmbH, Harxbütteler Str. 2, D-38110 Braunschweig, Germany.
} 


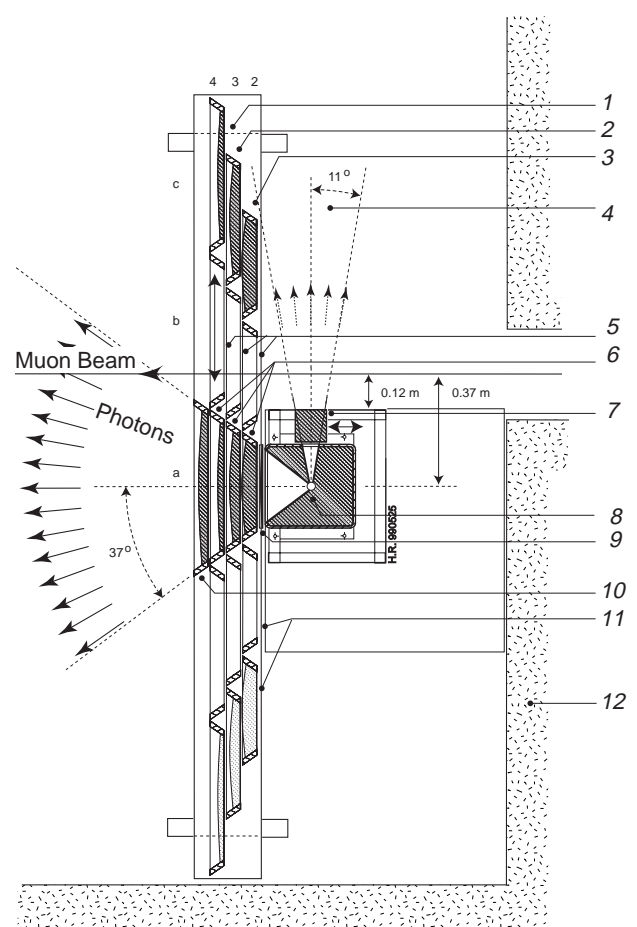

Figure 2: Horizontal cut of the source and of the set of lens-shaped lead filters which vary the actual photon rate. The absorption factor for the direct photons is the number quoted in later figures, and can range from 1 to 10,000 for the movable filters. 1-3: The three planes of movable filters. 4: Lateral outlet. 5: The central three filters consist of the collimator frame only, i.e. do not absorb. 6: The three filters in use. 7: Lateral outlet with shutter. 8: Lead block housing the source. 9: Thin fixed filter for angular correction. 10: Fixed filter to limit the max. photon flux. 11: Position of filters when moved to the left. 12: Concrete wall and floor.

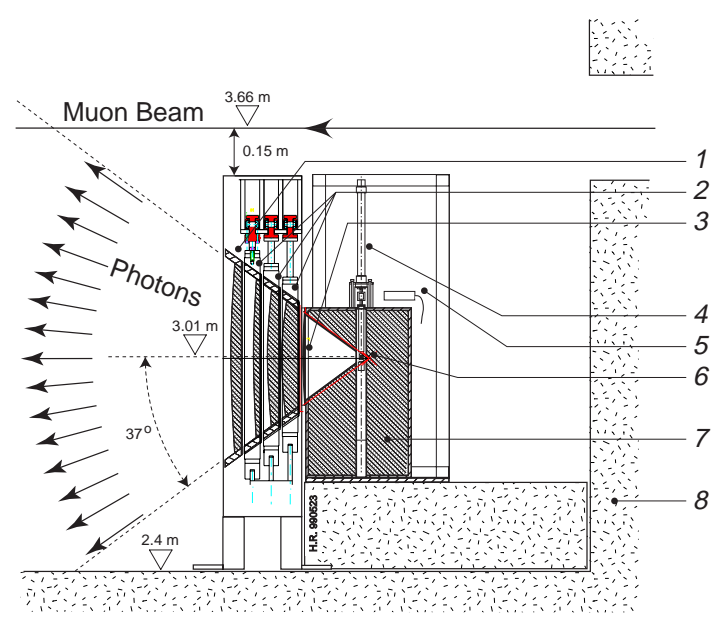

Figure 3: Vertical cut showing beam, source and filters. 1-3: Filters. 4: Rod with source at lower end is lifted to place the source in irradiation position. 5: Radiation monitor. 6: Source. 7: Lead housing of source. 8: Concrete. 


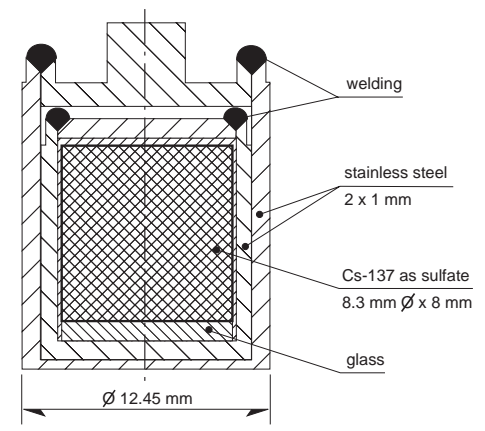

Figure 4: The actual source and its encasing.

on a $2 \mathrm{~mm}$ thick Aluminum support plate. The filter thickness is calculated at each point using $0.116 \mathrm{~mm}^{-1}$ for the absorption of the $662 \mathrm{keV}$ photons in lead, and taking into account the Aluminum plate with an absorption of $0.02 \mathrm{~mm}^{-1}$. Each movable filter is held in a $20 \mathrm{~mm}$ steel frame, shaped to extend the $74^{\circ} \times 74^{\circ}$ opening angle of the collimator.

The first filter has its shape calculated to transform the uniform rate on a spherical surface into a uniform rate on a planar surface. Its largest absorption factor is 2 , at the center. This angular correction filter is always present. The other filters have thus only to ensure an absorption factor which is independent of the direction of the incident photon.

A set of movable filters is installed in front of the source. It consists of three planes of three filters each (see Fig. 2). By remote control they can be moved laterally to place the desired filter of each plane in front of the source. The absorption factors of the filters are 100, 50, 1 for the plane closest to the source, 20, 10, 1 for the second plane, and 5, 2, 1 for the third plane, respectively. This gives 17 possible attenuation factors 1 (i.e. no filters), 2, 5, 10, 20, 40, 50, 100, 200, 250, 500, 1000, 2000, 2500, 4000, 5000 and 10000 .

Finally, as indicated in Fig. 2 a further filter can be attached in front of the system, to limit the largest photon rate, with the movable filters still providing the 17 possible attenuation factors below this. When quoting an absorption factor we give the combined absorption factor of the rate limiting filter and of the movable filters as an "ABS" factor; the factor from the angular correction filter is thus not included.

The absorption factors quoted above indicate which fraction of the monochromatic photons from the source ("direct" photons) will emerge from the filter. In addition, a comparable amount of scattered photons will also emerge. Their energy spectrum is broad. Finally, some photons backscattered from walls, floor, etc., will also reach the chamber. This means that the absorption factor used to construct the filter is only exact for the $662 \mathrm{keV}$ photons reaching the chamber; the actual hit rate depends on the geometry of the zone and on the (energy dependent) conversion probability in the chamber. However, the ratio of hit rates will follow closely the ratio of filter factors. Simulations and calibration data are given below.

\subsection{The beam}

The detectors in the Gamma Irradiation Facility are exposed simultaneously to photons from the Cesium source and to high energy particles from the X5 beam line, which is part of the West Area test beam complex at the CERN Super Proton Synchrotron. A beam of $450 \mathrm{GeV}$ protons is slowly extracted onto a primary target, consisting of a $400 \mathrm{~mm}$ long Beryllium plate. The typical duration of each extraction cycle is 2.38 seconds with a repetition period of 14.4 seconds. Hadrons and electrons emerging from this target are momentum selected and transported by the so-called H3 beam line to the X5 secondary target, located approximately $650 \mathrm{~m}$ downstream of the primary target. Normally pions and/or electrons produced in the X5 target are transported to the test zones at the end of the $200 \mathrm{~m}$ long X5 beam. If the X5 beam is tuned to a momentum exceeding $57 \%$ of the $\mathrm{H} 3$ beam momentum, muons from pion and 
kaon decays along the last part of the $\mathrm{H} 3$ beam will be transported along the $\mathrm{X} 5$ beam. The Gamma Irradiation Facility is located at the downstream end of the X5 beam line, just behind the final dump ( $2.4 \mathrm{~m}$ of iron followed by $1.6 \mathrm{~m}$ of concrete) which only muons penetrate. During typical operation of the West Area complex the muon momentum is around $100 \mathrm{GeV}$ and the flux about $10^{4} \mu^{-}$per SPS cycle, traversing a $10 \times 10 \mathrm{~cm}^{2}$ area. After traversal of the X5 dump, these muons are tagged by a scintillator and their impact position and angle measured by two delay wire chambers [9], separated by $2.4 \mathrm{~m}$, each having $200 \mu \mathrm{m}$ space resolution. A further beam chamber has been added downstream of the GIF zone to improve the measurement precision at the position of the detectors under test. Two pairs of scintillators, $3 \mathrm{~m}$ apart, provide a trigger signal with a timing precision better than $0.5 \mathrm{~ns}$.

On special request, the final dump of the X5 beam can be opened and a tertiary electron or pion beam of momentum between 5 and $200 \mathrm{GeV}$ can be transported into the Gamma Irradiation Facility. The electron beam is typically limited to $100 \mathrm{GeV}$. It is possible to provide $200 \mathrm{GeV}$ electrons as a secondary beam, but at the cost of being bound to run electrons of the same energy in the parallel beam line $\mathrm{X} 7$. The intrinsic momentum resolution of these beams is $0.8 \%$. Typical fluxes in the GIF range from $10^{2}$ to $10^{4}$ particles per pulse, with a RMS spot size down to about $1 \mathrm{~cm}^{2}$. This mode of operation allows calorimeters to be tested and calibrated in the presence of strong background radiations from the source.

Outside the scheduled running periods of the accelerator, a dedicated set of counters has been used to provide a trigger for cosmic rays traversing the detectors.

\subsection{Controls and safety}

The irradiator is controlled from a single console located in the irradiator control room next to the GIF area. All safety functions related to the zone are grouped in this console. This comprises search boxes, infrared detectors to signal presence inside the zone, smoke detectors, explosive gas detectors, emergency stops, doors not closed properly, compressed air pressure. Two radiation detectors - one close to the source and one far away - monitor continuously the radiation levels inside the zone and are connected to the irradiator control. Any detected anomaly forces the source back into its shielded position and vetoes any further extraction of the source into irradiation position. All changes of state are registered and logged by software and in case of malfunctioning alarms are sent to the central control room. A unique "Manual Veto" key defines the conditions for access or irradiation. An acoustical signal warns when the source is being opened. Light signals indicate the state of the installation. The source can only be brought into irradiation position after having executed the required safety procedures. All veto signals from the Gamma Irradiator are combined into a single contact which is then delivered to the central SPS Experimental Areas access control system. In case the final dump of the X5 beam is opened in order to provide electron or pion beams into the GIF, the access condition for the GIF is logically combined with the access conditions for the X5 beam areas in a standard way.

Automatic logging on files is also used for all other parameters of the source, of the muon beam settings and on the actual beam intensity sensed. It is a key feature for runs operated remotely or even in unattended mode, as needed for the very long aging tests and when the beam control is given to another zone upstream GIF.

\section{$3 \quad$ Radiation field}

\subsection{Simulations and measurements}

To provide the experimenters with reference data on the expected gamma flux, a series of simulations has been performed. They show how collimators, filters and surrounding walls modify the photon distributions in energy and angle.

\subsection{Direct and scattered photons}

First, the effect of a narrow beam of $662 \mathrm{keV}$ photons impinging on a single flat lead absorber was studied. In the simulation done with the code GEANT [10] for this simplified 
geometry, all emerging particles were counted, irrespective of energy or angle of emission; surrounding materials were ignored. In addition to the monochromatic "direct" photons, one sees that above $20 \mathrm{~mm}$ lead an almost equal number of scattered and direct photons emerges (Fig. 5). The scattered photons have a wide distribution in angle and energy, as shown for $30 \mathrm{~mm}$ lead in Fig. 6. In contrast to this illustration of the basic effect of a simple lead absorber, the simulation used for all subsequent figures and tables includes the full geometry of the source, filters, floor and walls.

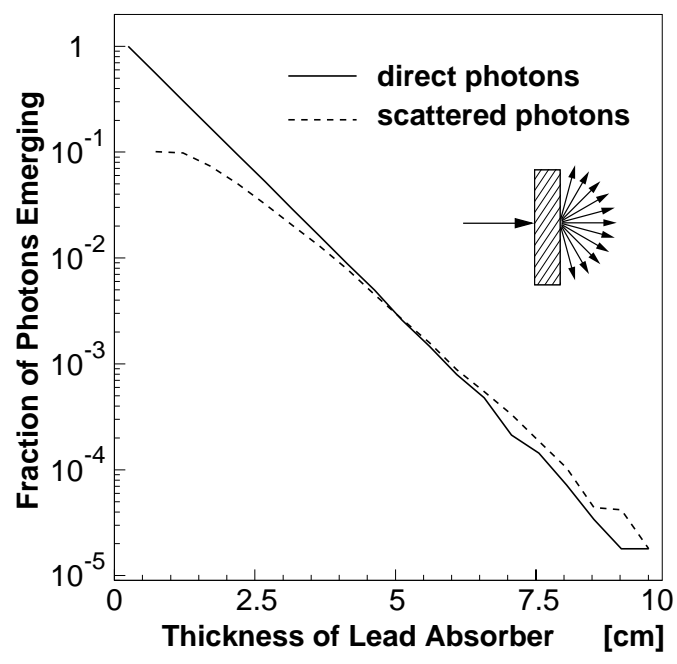

Figure 5: Fraction of the number of photons which emerge from a lead plate when $662 \mathrm{keV}$ photons impinge at normal incidence. The simulation was done for $10^{6}$ incident photons. In addition to the monochromatic direct photons (solid line), a comparable amount of scattered, softer photons also emerges (dashed line). The nominal absorption factor for the lead filters refers to the direct photons only.

\subsection{Source, filters and walls}

In a second step the details of the source, the shielding assembly geometry, the angular correction filter, three external moving filters and the GIF walls were entered in a simulation code (MCNP4b, [11]) and calculations were made for absorption factors 1, 2, 5, 10, 50, 100 and 500. The flux spectra, in free air, were simulated on a $20 \times 20 \mathrm{~cm}^{2}$ area at distances of 50,155 , 300 and $400 \mathrm{~cm}$ from the source along the beam axis, and normalized to the nominal activity of the source ( $740 \mathrm{GBq}, 0.85$ photon branching ratio).

As expected, the different materials around the source (holder, lead case, stainless steel collimators, lead filters, walls) spread the Cesium monoenergetic line by adding a low energy radiation. The result of the simulation for the contribution of the scattered photons to the total energy spectrum is shown in Fig. 7 for absorption factor 1 . The region due to filters and collimators extends from largest energies down to about $80 \mathrm{keV}$ for Compton scattering and hasa peak from $\mathrm{Pb}$ fluorescent $\mathrm{X}$-ray emission at $72 \mathrm{keV}$ ). The dashed area is an albedo region populating the lower energies and originating from Compton scattering off the walls. The relatively large number of scattered photons at absorption factor 1 reflects the fact that the collimators, the encapsulation of the Cesium and the filter for angular correction, which are always present, already represent a significant absorber thickness.

For photons emerging from the pointlike source, the flux per unit area decreases with the square of the distance from the source. However, it is interesting to note that the GIF walls create an albedo radiation that increases when moving away from the source. This is shown in Fig. 8 for an absorption factor 10: when moving away from the source along the beam axis 


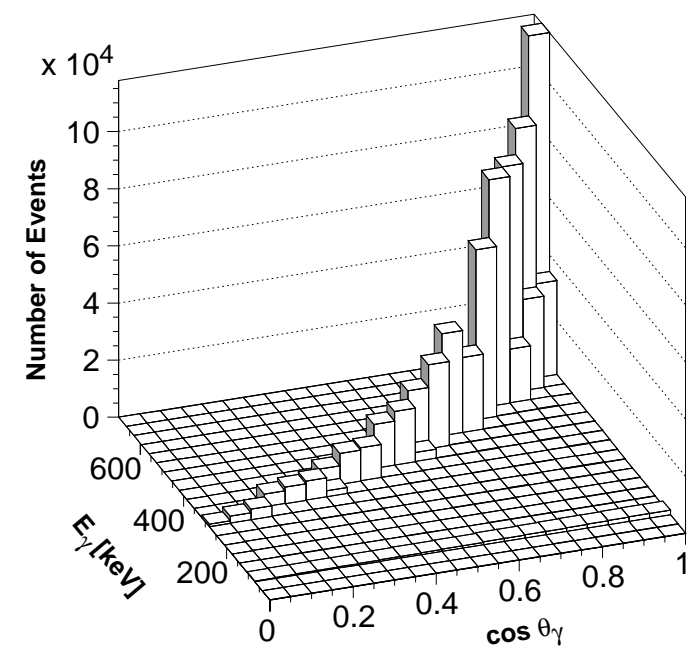

Figure 6: Distribution in angle and energy of the scattered photons emerging from a flat lead plate. The simulation was done for $10^{6}$ incident photons of $662 \mathrm{keV}$ and a $3 \mathrm{~cm}$ thick plate. The large peak of unscattered photons is not shown.

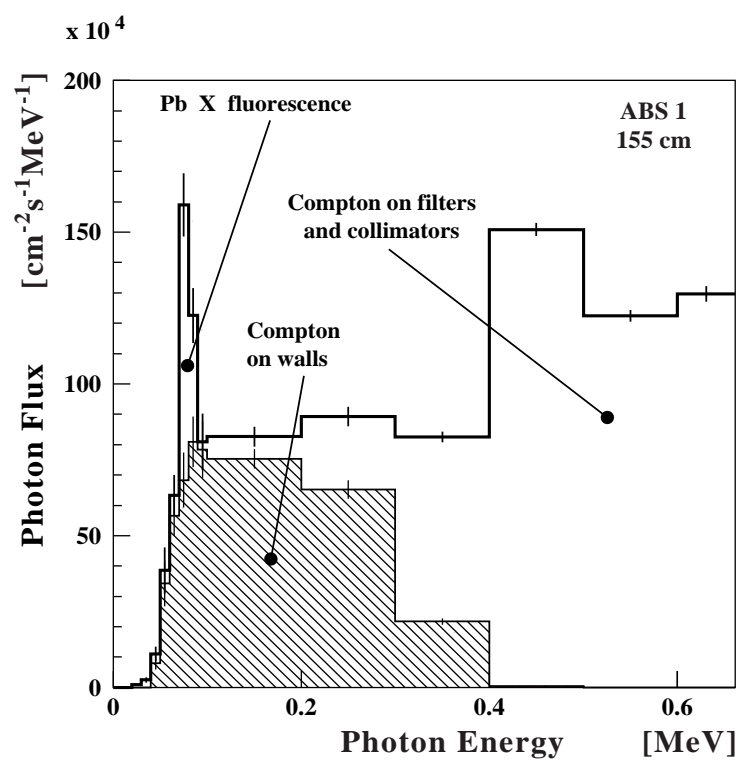

Figure 7: Simulated spectrum of the scattered photons at $155 \mathrm{~cm}$ from the source on the axis of the irradiation field and for absorption factor (ABS) 1. The three main contributions are indicated: two are from filters and collimators (white area), the other from walls and floor (dashed area). The total flux of scattered photons amounts to $6.4 \times 10^{5} \mathrm{~cm}^{-2} \mathrm{~s}^{-1}$, while the flux of direct photons (at $662 \mathrm{keV}$, not shown) is $8.0 \times 10^{5} \mathrm{~cm}^{-2} \mathrm{~s}^{-1}$ 
from $50 \mathrm{~cm}$ to $400 \mathrm{~cm}$, the albedo contribution changes from about $3 \%$ to $50 \%$ of the scattered photons.

Comparing different filter configurations shows that the contribution from scattered photons is expected to increase with absorption factor. The ratio of the number of scattered photons to direct photons ranges from $50 \%$ with absorption factor 1 to about $185 \%$ with absorption factor 100 at $50 \mathrm{~cm}$ from the source, and from $130 \%$ to about $330 \%$ at $400 \mathrm{~cm}$ from the source. It should be remembered, however, that this variation is quite small if compared with the corresponding factor 100 variation in the rate of direct photons, as already evident for the simple geometry from Fig. 5 .
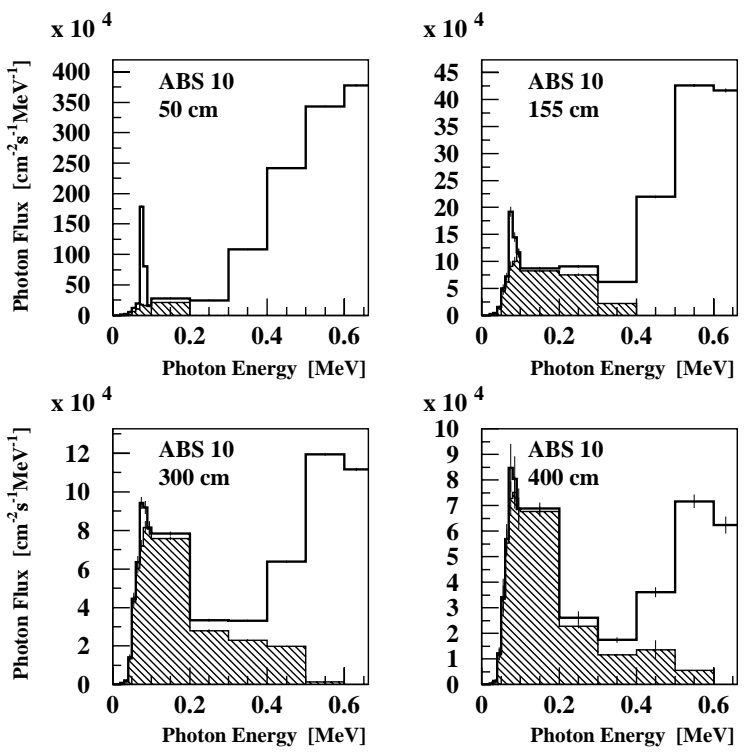

Figure 8: Dependence of the spectrum of scattered photons on the distance from the source. Spectra evaluated for absorption factor 10 and at 50, 155, 300 and $400 \mathrm{~cm}$ from the source along the beam axis. The dashed area in each plot indicates the radiation scattered off the walls. This albedo radiation turns out to be important at places more distant from the source but closer to the wall.

\begin{tabular}{|c|c|c|c|c|}
\hline \multicolumn{4}{|c|}{ ABS factors at $155 \mathrm{~cm}$ distance from the source } \\
\hline \hline Nominal & \multicolumn{2}{|c|}{ MCNP4B } & \multicolumn{2}{c|}{ Experimental } \\
ABS & Peak $(662 \mathrm{keV})$ & Total & IC10X & Film $^{a}$ \\
\hline 2 & $2.0 \pm 0.03$ & $1.8 \pm 0.02$ & $1.7 \pm 0.2$ & $1.9 \pm 0.2$ \\
5 & $5.0 \pm 0.1$ & $3.9 \pm 0.1$ & $3.6 \pm 0.4$ & $4.2 \pm 0.4$ \\
10 & $9.9 \pm 0.1$ & $7.4 \pm 0.1$ & $6.0 \pm 0.6$ & $8.1 \pm 0.8$ \\
50 & $47.1 \pm 0.7$ & $26.4 \pm 0.3$ & $23 \pm 2$ & \\
100 & $92 \pm 2$ & $48.3 \pm 0.6$ & $40 \pm 4$ & \\
500 & $471 \pm 24$ & $197 \pm 6$ & & \\
\hline
\end{tabular}

Table 1: Absorption factors simulated with the MCNP4b code compare well to the nominal value and to experimental data obtained with an IC10X ionization chamber and film dosemeters. Values are relative to those for absorption factor 1 . The first two columns refer to direct photons only. ${ }^{a}$ Film exposition was at $193 \mathrm{~cm}$.

In Tab. 1 absorption factors from simulation are confronted with nominal and experimental values. The simulation is given separately for direct photons, which corresponds to the nominal absorption factor, and for all photons, to compare with the experimental data. 
The experimental data were taken with an ionization chamber $^{3)}$ and with film dosemeters ${ }^{4)}$. This kind of ionization chamber and the films are sensitive to the total radiation and hence should be compared with the simulated total number of photons. The agreement in Tab. 1 is within the experimental errors.

As an additional test of the validity of the simulation the dose rate in air, as measured with the ionization chamber at $117 \mathrm{~cm}$ from the source was compared with the value obtained from the simulation with the MNCP4b code for a source activity of $740 \mathrm{GBq}$. The former resulted in $21 \pm 1 \mathrm{mGy} / \mathrm{h}$, in very good agreement with the calculated value of $22.1 \pm 0.5 \mathrm{mGy} / \mathrm{h}$.

Table 2 summarizes the predicted flux for a nominal activity of $740 \mathrm{GBq}$ at various distances from the source along the axis of the irradiation field. For each absorption factor the flux is given for both direct and total radiation.

\begin{tabular}{|c|c|c|c|c|}
\hline \multicolumn{5}{|c|}{ Direct radiation $\mathrm{E}_{\gamma}=662 \mathrm{keV}$} \\
\hline \multirow{2}{*}{$\begin{array}{c}\text { Nominal } \\
\text { ABS }\end{array}$} & \multicolumn{4}{|c|}{$\begin{array}{ll}\text { Photon flux }\left[\mathrm{cm}^{-2} \mathrm{~s}^{-1}\right]\end{array}$} \\
\hline & at $50 \mathrm{~cm}$ & at $155 \mathrm{~cm}$ & at $300 \mathrm{~cm}$ & at $400 \mathrm{~cm}$ \\
\hline 1 & $0.79 \cdot 10^{7} \pm 0.2 \%$ & $0.80 \cdot 10^{6} \pm 0.6 \%$ & $0.22 \cdot 10^{6} \pm 0.6 \%$ & $0.12 \cdot 10^{6} \pm 0.4 \%$ \\
\hline 2 & $0.39 \cdot 10^{7} \pm 0.3 \%$ & $0.40 \cdot 10^{6} \pm 1.0 \%$ & $0.11 \cdot 10^{6} \pm 1.0 \%$ & $0.60 \cdot 10^{5} \pm 0.7 \%$ \\
\hline 5 & $0.16 \cdot 10^{7} \pm 0.5 \%$ & $0.16 \cdot 10^{6} \pm 1.6 \%$ & $0.44 \cdot 10^{5} \pm 1.6 \%$ & $0.24 \cdot 10^{5} \pm 1.2 \%$ \\
\hline 10 & $0.79 \cdot 10^{6} \pm 0.1 \%$ & $0.81 \cdot 10^{5} \pm 0.4 \%$ & $0.22 \cdot 10^{5} \pm 0.4 \%$ & $0.12 \cdot 10^{5} \pm 0.3 \%$ \\
\hline 50 & $0.16 \cdot 10^{6} \pm 0.3 \%$ & $0.17 \cdot 10^{5} \pm 0.8 \%$ & $0.46 \cdot 10^{4} \pm 0.8 \%$ & $0.25 \cdot 10^{4} \pm 0.6 \%$ \\
\hline 100 & $0.81 \cdot 10^{5} \pm 0.4 \%$ & $0.87 \cdot 10^{4} \pm 1.1 \%$ & $0.23 \cdot 10^{4} \pm 1.1 \%$ & $0.13 \cdot 10^{4} \pm 0.9 \%$ \\
\hline 500 & $0.16 \cdot 10^{5} \pm 1.5 \%$ & $0.17 \cdot 10^{4} \pm 4.5 \%$ & $0.47 \cdot 10^{3} \pm 4.3 \%$ & $0.25 \cdot 10^{3} \pm 3.4 \%$ \\
\hline \multicolumn{5}{|c|}{$\begin{array}{c}\text { Total radiation } \mathrm{E}_{\gamma} \leq 662 \mathrm{keV} \\
\end{array}$} \\
\hline Nominal & \multicolumn{4}{|c|}{ Photon flux $\left[\mathrm{cm}^{-2} \mathrm{~s}^{-1}\right]$} \\
\hline ABS & at $50 \mathrm{~cm}$ & at $155 \mathrm{~cm}$ & at $300 \mathrm{~cm}$ & at $400 \mathrm{~cm}$ \\
\hline 1 & $0.12 \cdot 10^{8} \pm 0.2 \%$ & $0.14 \cdot 10^{7} \pm 0.5 \%$ & $0.45 \cdot 10^{6} \pm 0.5 \%$ & $0.28 \cdot 10^{6} \pm 0.5 \%$ \\
\hline 2 & $0.68 \cdot 10^{7} \pm 0.3 \%$ & $0.80 \cdot 10^{6} \pm 0.8 \%$ & $0.25 \cdot 10^{6} \pm 0.8 \%$ & $0.16 \cdot 10^{6} \pm 0.6 \%$ \\
\hline 5 & $0.31 \cdot 10^{7} \pm 0.4 \%$ & $0.36 \cdot 10^{6} \pm 1.2 \%$ & $0.11 \cdot 10^{6} \pm 1.2 \%$ & $0.70 \cdot 10^{5} \pm 0.9 \%$ \\
\hline 10 & $0.16 \cdot 10^{7} \pm 0.1 \%$ & $0.19 \cdot 10^{6} \pm 0.3 \%$ & $0.59 \cdot 10^{5} \pm 0.3 \%$ & $0.37 \cdot 10^{5} \pm 0.3 \%$ \\
\hline 50 & $0.44 \cdot 10^{6} \pm 0.2 \%$ & $0.53 \cdot 10^{5} \pm 0.5 \%$ & $0.16 \cdot 10^{5} \pm 0.5 \%$ & $0.10 \cdot 10^{5} \pm 0.4 \%$ \\
\hline 100 & $0.23 \cdot 10^{6} \pm 0.2 \%$ & $0.29 \cdot 10^{5} \pm 0.7 \%$ & $0.89 \cdot 10^{4} \pm 0.7 \%$ & $0.56 \cdot 10^{4} \pm 0.5 \%$ \\
\hline 500 & $0.54 \cdot 10^{5} \pm 0.9 \%$ & $0.71 \cdot 10^{4} \pm 2.4 \%$ & $0.22 \cdot 10^{4} \pm 2.3 \%$ & $0.14 \cdot 10^{4} \pm 1.9 \%$ \\
\hline
\end{tabular}

Table 2: Photon flux predicted with the MCNP4b code for a nominal ${ }^{137}$ Cs activity of $740 \mathrm{GBq}$ at various distances from the source along the axis of the irradiation field. For each absorption factor the flux is given for both direct and total radiation. Only statistical errors are given.

\subsection{Distribution over detector plane}

Measurements were performed to assess the uniformity of the photon flux over the surface of a detector, which represents a plane orthogonal to the axis of the irradiation field. The presence of scattered photons complicates the estimation of absolute rates, rate dependence on absorption factor, and rate dependence on distance, due to the energy dependence of the detector sensitivity. But their presence should have less impact on the uniformity over the detector plane.

In the following figures values are given for the photon flux measured and simulated along the two transverse (vertical and horizontal) directions orthogonal to the axis of the irradiation field. The experimental data were obtained with another ionization chamber ${ }^{5)}$ at $155 \mathrm{~cm}$ distance from the source, in 5 or $10 \mathrm{~cm}$ steps. The horizontal scan ranges from $-150 \mathrm{~cm}$ to $+150 \mathrm{~cm}$ from the field axis, while the vertical scan was limited by the floor and ranges from $-15 \mathrm{~cm}$ to $+150 \mathrm{~cm}$.

\footnotetext{
3) Type IC10X, from ROTEM-Industries Ltd., ROTEM Industrial Park, P.O. Box 9046, Beer-Sheva 84190, Israel

4) Badge type DFB-A, from X-Gammaguard, Via Gorizia 40, I-21047 Saronno (VA), Italy

5) Type 23361, from PTW-Freiburg, Lörracher Str. 7, D-79115 Freiburg, Germany
} 
The dose in air at each point was then normalized to the value at the central point. Care was taken to have the axis of the cylindrical ionization chamber of about $4 \mathrm{~cm}$ diameter oriented vertically for the horizontal scan, hence subtending the same cross section of particles flux at every angle. Correspondingly, the chamber axis was horizontal during the vertical scan. The simulation results are obtained for elements of $20 \times 20 \mathrm{~cm}^{-2} \mathrm{~s}^{-1}$ (or smaller) area with surface normal to the axis of the irradiation field, and of $4 \mathrm{~cm}$ thickness. The angular dependence should thus be similar for ionization chamber and simulation cells.

The results for the horizontal scan are shown in Fig. 9 for an absorption factor 1 . The peak at the origin is due to the presence of a brass screw at the center of the lead filters and is well reproduced by the simulation. The measured rate is very uniform over the whole width of the curve. The sharp edge on each side demonstrates the correct operation of the collimators. Simulation and real data are in agreement at a level of few percent except in the regions close to the collimators.

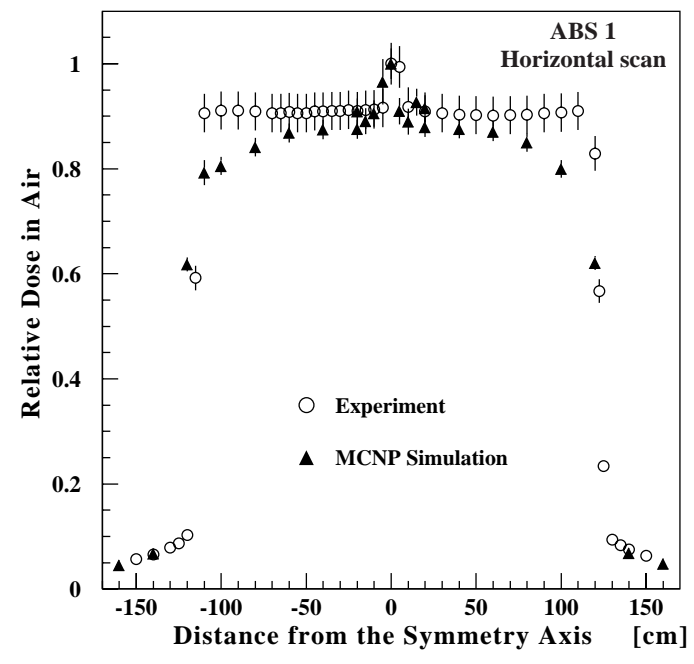

Figure 9: Horizontal scan, at $155 \mathrm{~cm}$ distance from the source and for absorption factor 1. Each point represents the dose in air normalized to the dose in air at the origin. The measurement (circles) is from the ionization chamber; the simulation (triangles) is for total radiation. The data show the desired uniform rate and the sharp edges defined by the collimators. The errors shown are statistical only.

The same features are observed in the case of the vertical scan, as evidenced by the curves shown in Fig. 10 and 11 for absorption factors 1 and 100. The somewhat better agreement between data and simulation in the case of the vertical scan suggests that the presence of further unaccounted material might have affected the horizontal scan. For absorption factor 100 the nonuniformity is more pronounced and at the same time the agreement between experimental and simulated data is better.

\section{Conclusion}

The Gamma Irradiation Facility (GIF) installed at CERN is a unique tool to test large area detectors under conditions of uniform and sustained high rates of random hits covering the full detector area, as will be encountered at the Large Hadron Collider. The photon energy of $0.662 \mathrm{MeV}$ used is also close to the average energy of about $2.5 \mathrm{MeV}$ expected for the LHC background. Muons for track calibration are provided by a high energy narrow beam of muons.

A large versatility is given by a system of lead filters which serve to make the photon rate nearly uniform over the detector plane, to limit the maximal rate, and above all to study rate dependent effects by varying the photon rates over up to four orders of magnitude via remote control. 


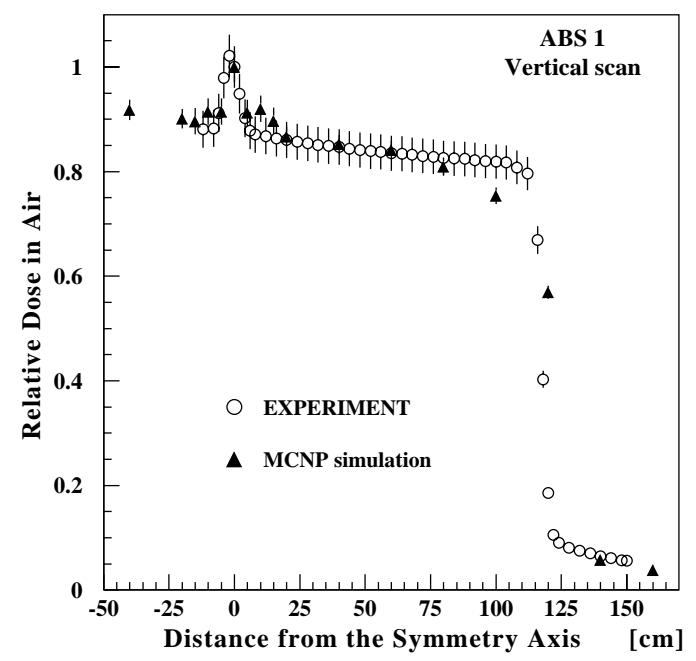

Figure 10: Vertical scan, at $155 \mathrm{~cm}$ distance from the source and for absorption factor 1.

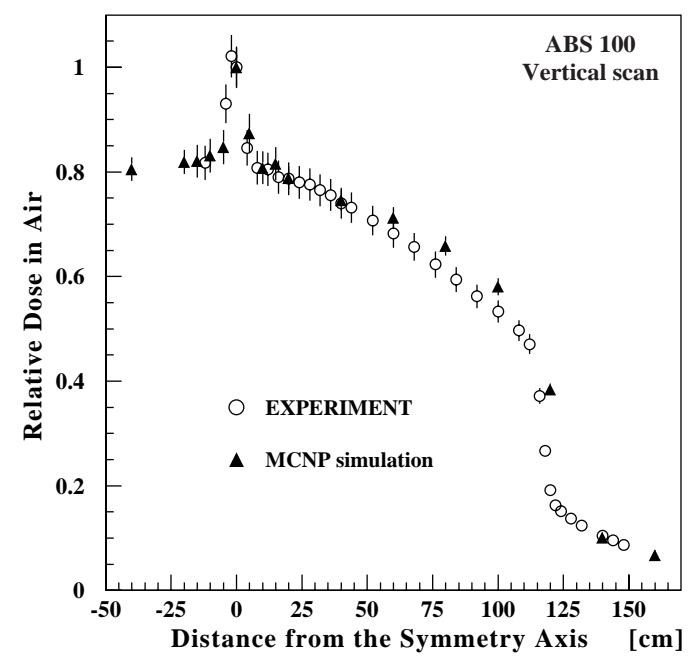

Figure 11: Vertical scan, at $155 \mathrm{~cm}$ distance from the source and for absorption factor 100 . 
The presence of massive objects like filters, walls, floor, equipment, is at the origin of scattered photons in addition to the original monochromatic "direct" photons. The scattered photons make simple precise predictions very difficult, since they will depend on details of the actual geometry and on an eventual spectral dependence of the detector response. Therefore it is adequate to quote the absorption factor of the filters with respect to the direct photons. Since the number of scattered photons is comparable to the number of direct photons over the wide range of absorption factors used, simple predictions can nevertheless be made to within a factor 2 .

With a series of measurements and calculations the main characteristics of the radiation field were studied. Experimental data demonstrate the uniformity of the photon rate over the plane of a detector and the correctness of the nominal filter factors. The simulation allowed to study the main contributions to the radiation field and to predict rates and spectra. Confrontation between experiment and simulation confirmed that the simulation is able to produce detailed and precise predictions.

Finally, the GIF is intensively serving all four LHC collaborations for performance and aging tests of large muon chambers under LHC conditions.

\section{$5 \quad$ Acknowledgements}

We would like to acknowledge the strong support given by CERN and in part by RWTH Aachen, which made the construction of this facility possible. Special thanks go to M. Clement, I. Crotty, J. Drecourt, M. Grill, C. Ferrari and T. Reynes for construction and installation of GIF; K. Bosseler, J. Grooten, K.D. Lohmann, F. Lutz, R. Pahlke, W. Reuter and J. Spanggaard for the filter system; L. Ulrici for help with the radiation measurements; A. Cojan and M. Clayton for software control; M. Hauschild, H. Taureg, M. Hoefert and G. Stevenson for safety-related discussions and authorisations, as well as to the members of the transport, electrical, gas and networking services at CERN. 


\section{References}

[1] The LHC Study Group: The Large Hadron Collider - Conceptual Design, CERN/AC/9505(LHC), 1995.

[2] CMS Collaboration: The Compact Muon Solenoid, Technical Proposal, CERN/LHCC 9438, 1994.

[3] ATLAS Collaboration: ATLAS Technical Proposal for a General-Purpose pp Experiment at the Large Hadron Collider at CERN, CERN/LHCC 94-43, 1994.

[4] CMS Collaboration: The Muon Project, Technical Design Report, CERN/LHCC 97-32, 1997.

[5] ATLAS

Collaboration: ATLAS Muon Spectrometer Technical Design Report, CERN/LHCC/9722, 1997.

[6] ALICE Collaboration: A Large Ion Collider Experiment, Technical Proposal, CERN/LHCC 95-71, 1995.

[7] ALICE Collaboration: Technical Design Report of the Dimuon Forward Spectrometer, CERN/LHCC 99-22 / ALICE TDR 5, 1994.

[8] LHCb Collaboration: A Large Collider Beauty experiment for precision measurements of CP-violation and rare decays, Technical Proposal, CERN/LHCC 98-4, 1998.

[9] A. Manarin and G. Vismara, The Delay Wire Chamber (DWC) description, CERN report LEP/BI-TA/Note 83-3, 1985.

[10] CERN Application Software Group: GEANT, Detector Description and Simulation Tool, Version 3.21, CERN Program library W5013.

[11] J.F. Briestmeister, Ed., MCNP - A General Monte Carlo N-Particle Transport Code, Los Alamos National Laboratories report LA-12625-M Version 4B, Los Alamos, New Mexico, 1997. 